\title{
Two separate mechanisms of enforced viral replication balance innate and adaptive immune activation
}

Namir Shaabania,b, ${ }^{\star}$, Vishal Khairnara,", Vikas Duhana, ${ }^{a}$, Fan Zhou ${ }^{a}$, Rita Ferrer Tura, Dieter Häussinger $^{\mathrm{b}}$, Mike Recher ${ }^{\mathrm{c}}$, Alexei V. Tumanov ${ }^{\mathrm{d}}$, Cornelia Hardta ${ }^{\mathrm{a}}$, Daniel Pinschewere ${ }^{\mathrm{e}}$ Urs Christen $^{\dagger}$, Philipp A. Lang, ${ }^{\text {, }}$, Nadine Honke ${ }^{a, b,{ }^{*}}$ and Karl S. Langa,b,

Running title: Lymphotoxin beta induces extracellular virus spread

*These authors contributed equally to this manuscript.

alnstitute of Immunology, Faculty of Medicine, University of Duisburg-Essen, Essen, Germany

bDepartment of Gastroenterology, Hepatology and Infectious Diseases, Heinrich Heine University Düsseldorf, Düsseldorf, Germany

cClinic for Primary Immunodeficiencies, Medical Outpatient Unit, and Immunodeficiency Laboratory, Department of Biomedicine, University Hospital Basel, Basel, Switzerland

'Trudeau Institute, Saranac Lake, NY, USA

eDepartment of Biomedicine - Haus Petersplatz, Division of Experimental Virology, University of Basel, Basel, Switzerland

${ }^{\dagger}$ Clinic of Goethe University Frankfurt, Frankfurt am Main, Germany

Address correspondence to KSL: karlsebastian.lang@uk-essen.de

Key words: immunopathology, lymphocytic choriomeningitis virus (LCMV), enforced viral replication 


\section{Abstract}

The induction of innate and adaptive immunity is essential for controlling viral infections. Limited or overwhelming innate immunity can negatively impair the adaptive immune response. Therefore, balancing innate immunity separately from activating the adaptive immune response would result in a better antiviral immune response. Recently, we demonstrated that Usp18-dependent replication of virus in secondary lymphatic organs contributes to activation of the innate and adaptive immune responses. Whether specific mechanisms can balance innate and adaptive immunity separately remains unknown. In this study, using lymphocytic choriomeningitis virus (LCMV) and replication-deficient single-cycle LCMV vectors, we found that viral replication of the initial inoculum is essential for activating virus-specific $\mathrm{CD}^{+} \mathrm{T}$ cells. In contrast, extracellular distribution of virus along the splenic conduits is necessary for inducing systemic levels of type I interferon (IFN-I). Although viral replication is driven primarily by Usp18, B cell-derived lymphotoxin beta contributes to the extracellular distribution of virus along the splenic conduits. Therefore, lymphotoxin beta regulates IFN-I induction independently of CD8+ T-cell activity. We found that two separate mechanisms act together in the spleen to guarantee amplification of virus during infection, thereby balancing the activation of the innate and adaptive immune systems. 


\section{Introduction}

During infection with a persistence-prone virus, type I interferon (IFN-I) and CD8 ${ }^{+} \mathrm{T}$ cells mediate the control of virus in infected cells. CD8 ${ }^{+} \mathrm{T}$ cells are activated early by viral replication; however, prolonged viral replication results in $\mathrm{CD}^{+} \mathrm{T}$-cell exhaustion and persistence of virus. Recently, striking data showed that IFN-I is like a yin and yang for controlling virus [1-3]. On the one hand, IFN-I is clearly antiviral, and it can influence the very early viral replication that can affect the course of persistent infection [4]. On the other hand, it induces programmed death ligand 1 (PD-L1) and interleukin IL-10 and thereby drives CD8 ${ }^{+} \mathrm{T}$-cell exhaustion $[1,2]$. Therefore limited production of IFN-I in the presence of highly activated CD8 ${ }^{+} \mathrm{T}$ cells results in severe immunopathology [5].

Lymphotoxins play an important role in several immunological processes, including lymph node development during embryogenesis, generation and activation of dendritic cells (DCs), and homeostatic control of chemokine expression in lymphoid organs [6-8]. Lymphotoxin beta (Ltb) plays a role in splenic architecture, developing conduits along the marginal zone and recruiting CD169+ macrophages $[9,10]$.

Recently we found that, during acute viral infection, viral replication in the marginal zone of the spleen and lymph nodes is essential for activating the innate and adaptive immune responses. This replication is dependent on ubiquitin-specific peptidase 18 (Usp18); therefore, the lack of Usp18 blunts the innate and adaptive immune responses [11, 12]. Although Ltb obviously enhances replication in secondary lymphatic organs [10], the interplay between Ltb and Usp18 remains unknown.

In the present study, using lymphocytic choriomeningitis virus (LCMV), we found that extracellular distribution of virus along the splenic conduits is necessary for inducing systemic levels of IFN-I but not for inducing virus-specific CD8 ${ }^{+} \mathrm{T}$ cells. Only the presence of Ltb-induced conduits allows the distribution of virus along the marginal zone; therefore, the lack of Ltb results in limited systemic production of IFN-I but in normal $\mathrm{CD} 8^{+} \mathrm{T}$-cell responses.

\section{Methods}

2.1. Mice: $P 14 / C D 45.1$ mice expressing a T-cell receptor (TCR) specific for LCMV glycoproteins 33 to 41 (LCMV-GP33-41) as a transgene were maintained on a C57BL/6 background and were used for adoptive transfer experiments [13]. Usp18 1 mice were generated in the Dong-ER 
Zhang laboratory (University of California san Diego, La jolla CA, USA) and were bred heterozygously on a Sv129 × C57BL/6 background, generation F4; these mice were directly compared with littermate control animals. Heavy chain joining gene null $\left(\mathrm{Jh}^{--}\right)$mice were maintained on a C57BL/6J background; lymphotoxin beta receptor null $\left(\mathrm{Ltbr}^{-\alpha}\right)$ mice were maintained on a C57BL/6N background. Conditionally targeted $L t b\left(L t b^{f / f t}\right)$ mice were crossed with CD19-Cre transgenic mice, and CD19-Cre-negative mice were used as littermate controls [14]. CD169-diphtheria toxin receptor (DTR) mice were maintained on a C57BL/6J background.

2.2. Virus and plaque assays: LCMV strain WE was originally obtained from $F$. LehmannGrube (Heinrich Pette Institute, Hamburg, Germany) and was propagated in L929 cells. Mice were infected intravenously with LCMV-WE at the indicated doses. Viral titers were measured with a plaque-forming assay using MC57 cells, as previously described [15]. We used recombinant LCMV (rLCMV; kindly provided by Daniel Pinschewer, University of Basel, Switzerland) to produce rLCMV vectors expressing a mutated version of LCMV-GP, which fails to confer viral infectivity but remains antigenic. We titrated these vectors according to standard procedures [16].

2.3. Lymphocyte transfer: Splenocytes from $P 14 / C D 45.1$ mice were injected intravenously into Usp18 $18^{-}$mice and littermate control mice. One day later, mice were infected with rLCMV $\left(6 \times 10^{4}\right.$ plaque-forming units [PFU]), and the percentage of P14/CD45.1 T cells in the spleen was assessed by flow cytometry.

2.4. Diphtheria toxin was purchased from Sigma (ST Louis, Missouri, USA) and was injected intraperitoneally on day -3 and day 2 at a dose of $30 \mu \mathrm{g} / \mathrm{kg}$.

2.5. Cell culture and generation of bone marrow-derived macrophages: Primary macrophages were generated by isolating bone marrow cells from femurs and tibias of mice. Macrophages were generated by culturing bone marrow cells in very low endotoxin Dulbecco's Modified Eagle's Medium (VLE-DMEM) supplemented with $10 \%(\mathrm{v} / \mathrm{v})$ fetal calf serum (FCS), $0.1 \%$ (v/v) $\beta$-mercaptoethanol ( $\beta$-ME), and $10 \mathrm{ng} / \mathrm{ml}$ macrophage colony-stimulating factor (M-CSF). After 9 days, macrophages were harvested for use in subsequent experiments. Recombinant mouse lymphotoxin $\alpha 2 / \beta 1$ was purchased from R\&D Systems (Minneapolis, USA).

2.6. Flow cytometry: Tetramers were provided by the National Institutes of Health $(\mathrm{NIH})$ Tetramer Core Facility (Emory University, Atlanta, GA, USA). Cells were stained with allophycocyanin (APC)-labeled GP33 major histocompatibility complex (MHC) class I tetramer (GP33/H-2Db) for 15 
minutes at $37^{\circ} \mathrm{C}$. After incubation, the samples were stained with anti-CD8 (eBiosciences, San Diego, USA) for 30 minutes at $4^{\circ} \mathrm{C}$. Absolute numbers of GP33-specific CD8 ${ }^{+} \mathrm{T}$ cells were calculated by fluorescence-activated cell sorting (FACS) analysis using fluorescent beads (BD Biosciences, San Jose, CA, USA). For measurement of intracellular IFN- $\gamma$, cells were stimulated with glycoprotein 33 (GP33) or nucleoprotein 396 (NP396), fixed with $2 \%$ formaldehyde for 10 minutes followed by permeabilization with saponin, and stained with anti-IFN- $\gamma$ or tumor necrosis factor (TNF- $\alpha$ ) antibodies (eBioscience). For MHC-I expression, Blood was stained on day 4 with anti CD8, anti CD4 and anti MHC-I (eBioscience).

2.7. ELISA: Enzyme-linked immunosorbent assays (ELISA) for IFN- $\alpha$ were performed according to the manufacturer's protocol (PBL Interferon Source, New Jersey, USA).

2.8. Histology: Conventional staining was performed as previously described [11]. Briefly, snap-frozen tissue was stained with rat anti-mouse polyclonal antibody to LCMV NP (VL4; made in house). Polyclonal anti-rat biotin antibody (eBioscience) and anti-biotin streptavidin peroxidase antibody (Thermo Scientific, Fremont, CA, USA) were then added before visualization with a 2solution diaminobenzidine (DAB) staining kit (Invitrogen, Carlsbad, CA, USA), after which the tissue was stained with hematoxylin. Immunofluorescence analyses of snap-frozen tissue were performed with anti-F4/80 (eBioscience), and anti-CD169 (Acris). Mouse nonspecific goat anti-guinea pig immunoglobulin (IgG; Jackson ImmunoResearch, West Grove, PA, USA) antibody was injected into mice to check in vivo the early distribution of the antibody.

\section{Results}

\subsection{Viral amplification is suppressed in peripheral organs but is allowed in spleen and lymph} nodes

During systemic infection, macrophages in the liver (Kupffer cells) capture most of the virus. This activity is observed when real time polymerase chain reaction (RT-PCR) for LCMV is performed 30 minutes after infection (Fig. 1A). If Kupffer cells are depleted with Clodronate-Liposome, the uptake of virus by the liver is reduced, whereas viral uptake by spleen, kidney and lung is increased (Fig. 1A). In normal case, without clodronate treatment, virus that is captured in the liver cannot amplify to detectable levels (Fig. 1B); however, virus can replicate only in spleen and lymph nodes

(Fig. 1B). Histologic analysis of the spleen demonstrates that virus spreads along the conduits of the 
marginal zone; thus, once a lymph follicle is infected, the virus is quickly distributed along the marginal zone in the spleen (Fig. 1C). These findings indicate that the spread of virus along marginal zone conduits is essential for viral replication in the spleen.

\subsection{Lack of lymphotoxin beta limits the flow along the marginal zone}

Next we questioned whether spread of the virus is regulated independently of viral replication and how these diverse mechanisms are regulated. We speculated that LTb is an important contributor to viral spread along the marginal zone; therefore, we infected wild-type (WT) and $L t b^{f / f /} C D 19-C r e$ (which lack LTb specifically on B cells) mice with LCMV and performed histologic analysis of the spleens. $L t t^{t / f i} C D 19-C r e$ mice exhibited several spots of infected cells but no distribution along the marginal zone (Fig. 2A). This finding suggests that Ltb is indeed necessary for viral distribution within the marginal zone of the spleen. We speculated that the lack of conduits in mice lacking the Ltb receptor $\left(\mathrm{Ltbr}^{-}\right)$limits flow along the marginal zone, a factor that could explain the limited spread of virus. To explore this speculation, we injected fluorescent beads into WT and $L t b r^{-}$mice. Histologic staining detected only limited numbers of beads in the marginal zone of $L t b r^{-}$mice compared to WT mice (Fig. 2B), a finding supporting the hypothesis that flow of the virus along the marginal zone of the spleen is limited in the absence of $L t b$.

To validate our finding we then injected mice with xenogenous nonspecific antibody labeled with fluorescent cyanine dye into WT and $L t b r^{-\alpha}$ mice and analyzed its distribution after 2 minutes. In WT mice, antibody stained the conduits along the marginal zone of the spleen (Fig. $2 \mathrm{C}$ ). In contrast, $L_{t b r^{-}}$mice exhibited limited staining along the marginal zone (Fig. 2C). Liver vessels were not affected by Ltb, a finding suggesting that the formation of conduits is specifically sensitive to Ltb (Fig. 2C) and are important for the cell to cell distribution of the viral particles. In conclusion, we found that the absence of Ltb limits flow of the antigen along the marginal zone, and this limitation in turn reduces the distribution of virus in the spleen.

\subsection{Usp18 and lymphotoxin beta allow viral replication in the spleen}

Next we questioned how the limited viral spread in the marginal zone influences the production of virus in the spleen. First, we infected bone marrow derived macrophages of WT and Usp18 deficient mice with LCMV and measured the virus titer in the supernatant after 24 hours. We 
found that Usp18 enhances LCMV replication on a cellular level, as demonstrated by in vitro study (Fig. 3A). Similarly, in vivo study showed that Usp18 enhances viral replication as WT mice have higher titer of LCMV relative to Usp18 $18^{-}$mice (Fig. 3B). In contrast, the lack of Ltb does not reduce viral replication in vitro (Fig. $3 C$ ). Moreover, treating cells with recombinant LTa2/ß1 did not reduce viral titer in the supernatant (Fig. 3C). Interestingly, In vivo, Ltbr ${ }^{-}$mice exhibit limited viral replication (Fig. 3D). To see whether this limited viral replication was due to reduction of CD169+ macrophages, we depleted CD169+ cells in CD169-DTR mice and infected them with LCMV. The depletion of $\mathrm{CD}_{169}+$ macrophages enhances viral replication (Fig. 3E and Supplementary Figure 1) which means that the limited replication in $L t b r^{-}$mice is not caused by the reduction in the number of CD169+ macrophages. Instead, viral replication is limited by Ltb-derived B cells, because viral replication is similarly reduced in $\mathrm{Jh}^{-/}$and $L t b^{f / f /} \mathrm{CD} 19-\mathrm{Cre}$ mice (Fig. 3F). Therefore we conclude that both Usp18 and $L t b$ are essential for enforced viral replication in the spleen.

\subsection{Usp18 and lymphotoxin beta are essential for inducing systemic type I interferon production, but only Usp18 influences CD8 ${ }^{+}$T-cell priming}

We found that Usp18 influences viral replication, whereas Ltb guarantees viral spread along the marginal zone. Both mechanisms are necessary for viral replication in the spleen. To determine how Usp18 and Ltb influence the innate and adaptive immune responses, we infected Usp $18^{-\sim}$ mice with 200 PFU of LCMV and measured IFN-I in the serum. We found that early viral replication is essential for IFN-I production (Fig. 4A). Similarly, IFN-I production is strongly impaired in $L t b^{f / f i} \mathrm{CD} 19$ Cre mice (Fig. 4B). Although viral replication was not completely inhibited in both Usp18 1 and $L t b^{f / f / I} \mathrm{CD} 19-\mathrm{Cre}$ mice, this replication was not enough to trigger IFN-I production. Next we wanted to examine the effect of Usp18 and $L t b$ on adaptive immune responses. As expected, the lack of Usp18 limits the magnitude of CD8+ T-cell responses (Fig. 4C). Lately, it was shown that IFN-I can protect $\mathrm{CD}^{+} \mathrm{T}$ cells from killing by NK cells through upregulation of MHC-I [17]. We wondered whether the reduction of $\mathrm{CD}^{+} \mathrm{T}$ cell numbers was due to lack of IFN-I production. To check this, we measured MHC-I expression on T cells on day 4 after infection. We found that Usp18-deficient T cells showed higher MHC-I expression than WT T cells (Supplementary Figure 2) which can be explained due to a stronger IFN-I signaling in these cells. 


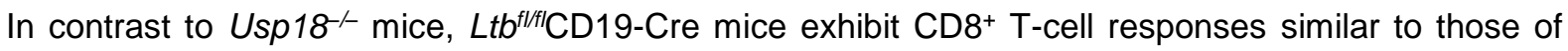
WT mice (Fig. 4D). This finding suggests that viral spread along the marginal zone is essential for IFN-I production but not for CD8+ T-cell priming.

\subsection{Extracellular spread of virus is essential for inducing systemic type I interferon production but not for inducing $\mathrm{CD}^{+} \mathrm{T}$-cell responses}

To determine whether the spread of virus along the marginal zone is essential for activating the innate but not the adaptive immune response, we used a single-cycle virus (rLCMV). Because this virus does not express the full LCMV-GP, cells infected with rLCMV do not produce infectious virus particles. However, viral genome transcription and replication are allowed within the cells that were initially infected. We infected C57BL/6 mice with LCMV or rLCMV. We found that, LCMV induces large amounts of serum IFN-I at almost all infectious doses tested, whereas rLCMV does not induce detectable levels of systemic IFN-I (Fig. 5A). Therefore, we conclude that the spread of virus is essential for inducing IFN-I.

We next examined the role of rLCMV in activating CD8 ${ }^{+} \mathrm{T}$ cells. We found that, compared to LCMV infection, infection with the rLCMV vector induces weaker but still potent virus-specific CD8 ${ }^{+} \mathrm{T}^{-}$ cell responses (Fig. 5B). As few as 100 infectious units produce measurable CD8 $8^{+}$T-cell responses. Immunization with $10^{5} \mathrm{PFU}$ of vector results in frequencies of epitope-specific CD8 ${ }^{+} \mathrm{T}$ cells that are similar to those produced by WT virus (Fig. 5B). To check whether single-cycle rLCMV exhibits limited CD8+ T-cell expansion in the absence of Usp18, we transferred splenocytes from P14/CD45.1 mice into WT and Usp18 ${ }^{-}$mice, These mice express a T-cell receptor (TCR) specific for LCMV glycoproteins 33 to 41 (LCMV-GP33-41), we infected both groups with rLCMV and measured the number of transferred CD8 ${ }^{+} \mathrm{T}$ cells. We found that $\mathrm{CD} 8^{+} \mathrm{T}$ cells expand much less in the absence of Usp18 compared to WT mice (Fig. 5C). These findings led us to conclude that the induction of systemic IFN-I depends on extracellular transfer and dissemination of virus. In contrast, replication of the initial virus inoculum is sufficient for inducing the production of virus-specific CD8+ $T$ cells.

\section{Discussion}

The results of this study show that both Usp18 and Ltb contribute to viral replication in the spleen. Whereas Usp18 directly influences virus replication, Ltb guarantees the spread of LCMV 
along the marginal zone. Although LCMV replication is sufficient for CD8 ${ }^{+}$T-cell activation, both effects, i.e., virus replication and extracellular virus transfer, are necessary for inducing systemic IFN-I.

These two mechanisms of virus propagation may influence the outcome of viral infection. This possibility is of special interest because both mechanisms influence replication separately. Usp18 is strongly induced by IFN-I, whereas Ltb is produced constantly in the spleen and is mainly influenced by the number of $B$ cells in a lymph follicle. Therefore, various infections could regulate both mechanisms separately. In the presence of B cells and high levels of Ltb, IFN-I is induced more efficiently.

We found that the spread of virus along the marginal zone is essential for the induction of systemic IFN-I. During LCMV infection, plasmacytoid dendritic cells (pDCs) produce large amounts of IFN-I $[18,19]$, for which the IFN-I-activating receptor retinoic acid-inducible gene-I (RIG-I) is at least partially responsible [20]. We determined that B cell-derived Ltb is an important contributor to extracellular distribution of virus along the marginal zone. However, it remained unclear why this distribution of virus is essential for IFN-I induction. One explanation could be that only large amounts of viral RNA can sufficiently activate pDCs. A second explanation could be that the composition of the cell membrane of infected cells along the marginal zone is different from that of other cell types; therefore, virus particles received from these cells could be more likely to infect pDCs. Indeed, recent findings show that the composition of the cell membrane can influence viral tropism [21]. A third explanation could be that this distribution of virus is important for infecting cells that are near pDCs. Consequently, those pDCs may sense the presence of infected cells and produce IFN-I. Recent findings have shown that uninfected $\mathrm{pDCs}$ can produce IFN-I by sensing infected cells; this mechanism is independent of intrinsic viral replication in pDCs [22]. However, more studies are needed for determining which of these explanations may be correct.

It remains unclear why $\mathrm{CD}^{+} \mathrm{T}$ cells but not IFN- $\alpha-$ producing cells can be activated by a single-cycle virus. We consider that the amount of viral protein and viral RNA within an infectious particle is limited compared to that in a virus-infected cell. Therefore, if one infectious rLCMV particle infects a DC, this virus-infected DC can probably generate a sufficient virus-specific $\mathrm{CD}^{+} \mathrm{T}$-cell response. 
A recent publications reported that an overwhelming IFN-I response can induce IL-10 and PD-L1, which then affect the exhaustion of $C D 8^{+} T$ cells $[1,2]$. Another supporting study showed a negative role of IFN- $\beta$ in controlling persistent viral infection [3]. In light of our findings, we suggest that inhibiting the extracellular spread of virus may limit the virus-induced production of IFN-I and thereby may prevent the exhaustion of $\mathrm{CD}^{+} \mathrm{T}$ cells. Various antiviral drugs target separate virus pathways. We suggest that antiviral drugs, which allow viral replication but block the release of virus particles, may inhibit viral propagation but still allow the priming and activation of antiviral $\mathrm{CD} 8^{+} \mathrm{T}$ cells. If so, treatment with these drugs may provide the most effective long-term protection against virus.

Further studies are needed to be done to show whether these two mechanisms may influence additionally the peripheral IFN-II production and thereby contribute to relapse of lupus erythematosus $[23,24]$

In conclusion, we found that viral replication and extracellular viral spread are two separate mechanisms of enforced viral replication. Whereas viral replication is essential for activation of the innate and adaptive immune systems, extracellular viral spread primarily affects systemic IFN-I production.

\section{Ethics Statement}

All experiments were performed with the animals housed in single ventilated cages. Experiments were conducted under the authorization of the Veterinäramt Nordrhein Westfalen (Düsseldorf, Germany) and in accordance with the German law for animal protection or the institutional guidelines of the Ontario Cancer Institute. The Landesamt für Natur, Umwelt und Verbraucherschutz Nordrhein-Westfalen (LANUV; State Agency for Nature, Environment, and Consumer Protection) approved all experiments (project numbers 84-02.04.2014.A242 and 8402.04.2012.A094).

\section{Acknowledgments}

We thank Konstanze Schättel and Patricia Spieker for technical support and Masato Tanaka for providing CD169-DTR mice. 


\section{References}

1. Teijaro, J.R., Ng, C., Lee, A.M., Sullivan, B.M., Sheehan, K.C., Welch, M., Schreiber, R.D., de la Torre, J.C., Oldstone, M.B. 2013. Persistent LCMV infection is controlled by blockade of type I interferon signaling. Science, 340: 207-11.

2. Wilson, E.B., Yamada, D.H., Elsaesser, H., Herskovitz, J., Deng, J., Cheng, G., Aronow, B.J., Karp, C.L., Brooks, D.G. 2013. Blockade of chronic type I interferon signaling to control persistent LCMV infection. Science, 340: 202-7.

3. Ng, C.T., Sullivan, B.M., Teijaro, J.R., Lee, A.M., Welch, M., Rice, S., Sheehan, K.C., Schreiber, R.D., Oldstone, M.B. 2015. Blockade of interferon Beta, but not interferon alpha, signaling controls persistent viral infection. Cell Host Microbe, 17: 653-61.

4. Sullivan, B.M., Teijaro, J.R., de la Torre, J.C., Oldstone, M.B. 2015. Early virus-host interactions dictate the course of a persistent infection. PLoS Pathog, 11: e1004588.

5. Lang, K.S., Georgiev, P., Recher, M., Navarini, A.A., Bergthaler, A., Heikenwalder, M., Harris, N.L., Junt, T., Odermatt, B., Clavien, P.A. et al. 2006. Immunoprivileged status of the liver is controlled by Toll-like receptor 3 signaling. J Clin Invest, 116: 2456-63.

6. Rennert, P.D., James, D., Mackay, F., Browning, J.L., Hochman, P.S. 1998. Lymph node genesis is induced by signaling through the lymphotoxin beta receptor. Immunity, 9: 71-9.

7. McCarthy, D.D., Summers-Deluca, L., Vu, F., Chiu, S., Gao, Y., Gommerman, J.L. 2006. The lymphotoxin pathway: beyond lymph node development. Immunol Res, 35: 41-54.

8. Kabashima, K., Banks, T.A., Ansel, K.M., Lu, T.T., Ware, C.F., Cyster, J.G. 2005. Intrinsic lymphotoxin-beta receptor requirement for homeostasis of lymphoid tissue dendritic cells. Immunity, 22: 439-50.

9. Futterer, A., Mink, K., Luz, A., Kosco-Vilbois, M.H., Pfeffer, K. 1998. The lymphotoxin beta receptor controls organogenesis and affinity maturation in peripheral lymphoid tissues. Immunity, 9: 59-70.

10. Moseman, E.A., lannacone, M., Bosurgi, L., Tonti, E., Chevrier, N., Tumanov, A., Fu, Y.X., Hacohen, N., von Andrian, U.H. 2012. B cell maintenance of subcapsular sinus macrophages protects against a fatal viral infection independent of adaptive immunity. Immunity, 36: 41526.

11. Honke, N., Shaabani, N., Cadeddu, G., Sorg, U.R., Zhang, D.E., Trilling, M., Klingel, K., Sauter, M., Kandolf, R., Gailus, N. et al. 2012. Enforced viral replication activates adaptive immunity and is essential for the control of a cytopathic virus. Nat Immunol, 13: 51-7.

12. Honke, N., Shaabani, N., Zhang, D.E., lliakis, G., Xu, H.C., Haussinger, D., Recher, M., Lohning, M., Lang, P.A., Lang, K.S. 2013. Usp18 driven enforced viral replication in dendritic cells contributes to break of immunological tolerance in autoimmune diabetes. PLoS Pathog, 9: e1003650.

13. Pircher, H., Burki, K., Lang, R., Hengartner, H., Zinkernagel, R.M. 1989. Tolerance induction in double specific T-cell receptor transgenic mice varies with antigen. Nature, 342: 559-61.

14. Tumanov, A., Kuprash, D., Lagarkova, M., Grivennikov, S., Abe, K., Shakhov, A., Drutskaya, L., Stewart, C., Chervonsky, A., Nedospasov, S. 2002. Distinct role of surface lymphotoxin expressed by B cells in the organization of secondary lymphoid tissues. Immunity, 17: 23950.

15. Lang, P.A., Contaldo, C., Georgiev, P., El-Badry, A.M., Recher, M., Kurrer, M., CervantesBarragan, L., Ludewig, B., Calzascia, T., Bolinger, B. et al. 2008. Aggravation of viral hepatitis by platelet-derived serotonin. Nat Med, 14: 756-61.

16. Flatz, L., Hegazy, A.N., Bergthaler, A., Verschoor, A., Claus, C., Fernandez, M., Gattinoni, L., Johnson, S., Kreppel, F., Kochanek, S. et al. 2010. Development of replication-defective lymphocytic choriomeningitis virus vectors for the induction of potent CD8+ T cell immunity. Nat Med, 16: 339-45. 
17. Xu, H.C., Grusdat, M., Pandyra, A.A., Polz, R., Huang, J., Sharma, P., Deenen, R., Kohrer, K., Rahbar, R., Diefenbach, A. et al. 2014. Type I interferon protects antiviral CD8+ T cells from NK cell cytotoxicity. Immunity, 40: 949-60.

18. Lang, P.A., Recher, M., Honke, N., Scheu, S., Borkens, S., Gailus, N., Krings, C., Meryk, A., Kulawik, A., Cervantes-Barragan, L. et al. 2010. Tissue macrophages suppress viral replication and prevent severe immunopathology in an interferon-I-dependent manner in mice. Hepatology, 52: 25-32.

19. Scheu, S., Dresing, P., Locksley, R.M. 2008. Visualization of IFNbeta production by plasmacytoid versus conventional dendritic cells under specific stimulation conditions in vivo. Proc Natl Acad Sci U S A, 105: 20416-21.

20. Zhou, S., Cerny, A.M., Zacharia, A., Fitzgerald, K.A., Kurt-Jones, E.A., Finberg, R.W. 2010. Induction and inhibition of type I interferon responses by distinct components of lymphocytic choriomeningitis virus. J Virol, 84: 9452-62.

21. Schneider-Schaulies, J. 2000. Cellular receptors for viruses: links to tropism and pathogenesis. J Gen Virol, 81: 1413-29.

22. Wieland, S.F., Takahashi, K., Boyd, B., Whitten-Bauer, C., Ngo, N., de la Torre, J.C., Chisari, F.V. 2014. Human plasmacytoid dendritic cells sense lymphocytic choriomeningitis virusinfected cells in vitro. J Virol, 88: 752-7.

23. Shaabani, N., Honke, N., Dolff, S., Gorg, B., Khairnar, V., Merches, K., Duhan, V., Metzger, S., Recher, M., Barthuber, C. et al. 2015. IFN-gamma licenses CD11b(+) cells to induce progression of systemic lupus erythematosus. J Autoimmun, 62: 11-21.

24. Hodge, D.L., Berthet, C., Coppola, V., Kastenmuller, W., Buschman, M.D., Schaughency, P.M., Shirota, H., Scarzello, A.J., Subleski, J.J., Anver, M.R. et al. 2014. IFN-gamma AU-rich element removal promotes chronic IFN-gamma expression and autoimmunity in mice. $J$ Autoimmun, 53: 33-45. 


\section{Figure legends}

Fig. 1: Viral amplification is suppressed in peripheral organs but allowed in spleen and lymph nodes.

(A) C57BL/6 mice were injected with empty liposomes (as controls) or were depleted of macrophages with clodronate liposomes. After one day, mice were infected with lymphocytic choriomeningitis virus (LCMV; $2 \times 10^{6}$ plaque-forming units [PFU]), and after 30 minutes copies of LCMV were quantified by real time polymerase chain reaction (RT-PCR) $(n=6)$. (B) C57BL/6 mice were infected with LCMV (200 PFU), and viral titers were determined in various organs at indicated time points $(n=4)$. (C) Histologic staining for LCMV nucleoprotein (NP) was performed on splenic sections from C57BL/6 mice infected intravenously with LCMV (200 PFU) for three days ( $n=3$; scale bar in main images, 500 $\mu \mathrm{m}$; inlets, $100 \mu \mathrm{m})$.

Fig. 2: Lack of lymphotoxin beta limits flow along the marginal zone.

(A) Results of conventional histologic analysis of splenic sections from wild-type (WT) mice and conditionally targeted lymphotoxin beta $\left(L t b^{t / f t}\right)$ CD19-Cre mice infected with lymphocytic choriomeningitis virus strain WE (LCMV-WE; 200 plaque-forming units [PFU]) for 3 days. LCMV, brown ( $\mathrm{n}=3$; scale bar main images, $500 \mu \mathrm{m}$; inlets, $100 \mu \mathrm{m}$ ). (B) Immunofluorescent staining of spleen sections from C57BL/6 mice 10 min after being injected with fluorescent beads. Beads, green; CD169, red; F4/80, blue ( $\mathrm{n}=3$; scale bar, $100 \mu \mathrm{m}$ ). (C) Immunofluorescent staining of spleen and liver sections from WT and lymphotoxin beta receptor deficient $\left(\mathrm{Ltbr}^{-}-\right)$mice 2 min after injection with antiguinea pig immunoglobulin (IgG), red ( $n=5$; scale bar, $100 \mu m)$.

Fig. 3: Ubiquitin-specific peptidase 18 and lymphotoxin beta allow viral replication in the spleen.

(A) Bone marrow-derived macrophages from ubiquitin-specific peptidase 18 deficient (Usp18--) mice and littermate control mice were infected with lymphocytic choriomeningitis virus strain WE (LCMVWE; multiplicity of infection [MOI], 1). After 24 hours, viral titer in the supernatant was determined $(\mathrm{n}=4)$. (B) Usp18 ${ }^{-}$and littermate control mice were infected intravenously with LCMV-WE (200 plaque-forming units [PFU]). After 3 days, viral titers in the spleen were determined $(n=3)$. (C) Bone marrow-derived macrophages from wild-type (WT) and lymphotoxin beta receptor deficient $\left(L t b r^{-}\right)$ mice were infected with LCMV-WE (MOI, 1) and treated with interferon alpha (IFN- $\alpha$; $50 \mathrm{U} / \mathrm{ml}$ ), 
(lymphotoxin $\alpha 2 / \beta 1 ; 100 \mathrm{ng} / \mathrm{ml}$ ), or both, or were left untreated. After 24 hours, viral titer in the supernatant was determined ( $\mathrm{n}=3-6)$. (D) WT and $L t b r^{-}$- mice were infected intravenously with LCMVWE (200 PFU). After 3 days, viral titers in the spleen were determined ( $n=3-4)$. (E) C57/BL6 or CD169 diphtheria toxin receptor (CD169-DTR) mice were treated with diphtheria toxin and infected with LCMV (200 PFU). After 2 days, viral titers in the spleen were determined $(n=5)$. (F) Heavy chain joining gene deficient $\left(\mathrm{Jh}^{-/}\right)$, conditionally targeted Ltb $\left(L t b^{f / / f}\right)$ CD19-Cre, and control mice were infected intravenously with LCMV-WE (200 PFU). After 3 days, viral titers in the spleen were determined $(n=3-4)$.

Fig. 4: Ubiquitin-specific peptidase 18 and lymphotoxin beta are essential for inducing systemic type I interferon, but only ubiquitin-specific peptidase 18 influences the priming of CD8+ $T$ cells.

(A) Ubiquitin-specific peptidase 18 deficient (Usp18--) mice and littermate control mice were infected intravenously with 200 PFU of lymphocytic choriomeningitis virus (LCMV). Interferon alpha (IFN- $\alpha$ ) levels in serum were determined by enzyme-linked immunosorbent assay (ELISA) at the indicated time points $(\mathrm{n}=4)$. (B) Wild-type (WT) and conditionally targeted lymphotoxin beta $\left(L t b^{f / / f}\right)$ CD19-Cre mice were infected intravenously with 200 plaque-forming units (PFU) of LCMV strain WE. IFN- $\alpha$ levels were measured in the serum by ELISA at the indicated time points $(n=3-7)$. (C) Fluorescenceactivated cell sorting (FACS) analysis of $\mathrm{CD}^{+} \mathrm{T}$ cells positive for glycoprotein (GP33), nucleoprotein 396 (NP396), and IFN- $\gamma$ in splenocytes from Usp18 ${ }^{-}$and littermate control mice on day 30 after infection with 200 PFU LCMV-WE and six hours after restimulation with GP33 peptide or NP396 peptide $(n=4)$. (D) FACS analysis of $\mathrm{GP}_{3}{ }^{+} \mathrm{IFN}-\mathrm{\gamma}^{+} \mathrm{CD} 8^{+} \mathrm{T}$ cells and GP33+ tumor necrosis factor TNF- $\alpha^{+} C D 8^{+} T$ cells in splenocytes from $L t b^{f / f i} C D 19-C r e$ mice and control mice on day 14 after infection with 200 PFU LCMV-WE and six hours after restimulation with GP33 peptide $(n=5-8)$. n.s., not statistically significant.

Fig. 5: Extracellular spread of virus is essential for inducing systemic type I interferon but not for inducing a $\mathrm{CD} 8^{+} \mathrm{T}$-cell response.

(A) C57BL/6 mice were infected intravenously with the indicated doses of lymphocytic choriomeningitis virus strain WE (LCMV-WE) or recombinant LCMV (rLCMV). Interferon alpha (IFN- $\alpha)$ in the serum was measured by enzyme-linked immunosorbent assay (ELISA) at the indicated time 
points $(n=3)$. (B) Percentages of nucleoprotein 396 (NP396)-specific and glycoprotein 33 (GP33)specific CD8 ${ }^{+} \mathrm{T}$ cells in blood were determined on day 9 after infection with the indicated doses of LCMV or rLCMV $(n=3-5)$. (C) We adoptively transferred $10^{7}$ splenocytes from P14/CD45.1 mice into Ubiquitin-specific peptidase 18 deficient (Usp18--) or wild-type (WT) littermate control mice. After 24 hours, mice were infected intravenously with $\operatorname{rLCMV~}\left(6 \times 10^{4}\right.$ plaque-forming units [PFU]). Absolute number of CD45.1+ CD8 ${ }^{+}$T cells in total lymphocytes in blood and spleen was determined ( $\left.n=3-6\right)$. 
Figure 1

A

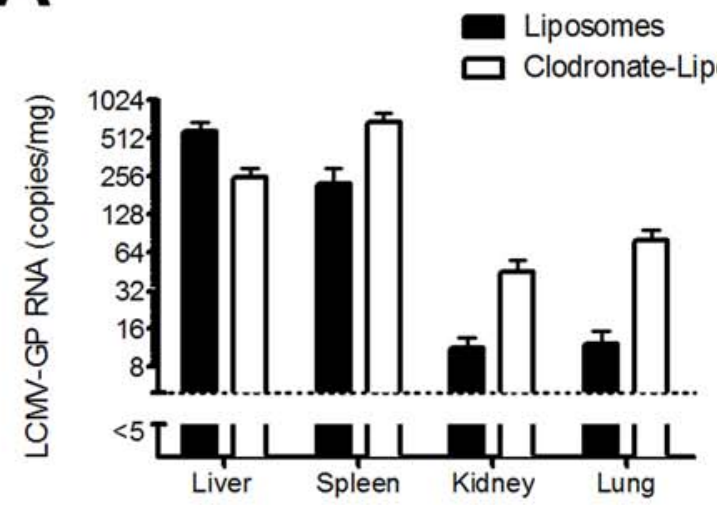

C

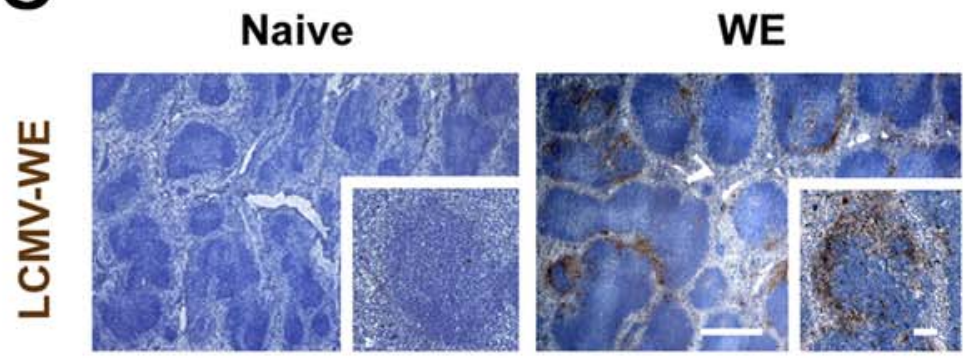

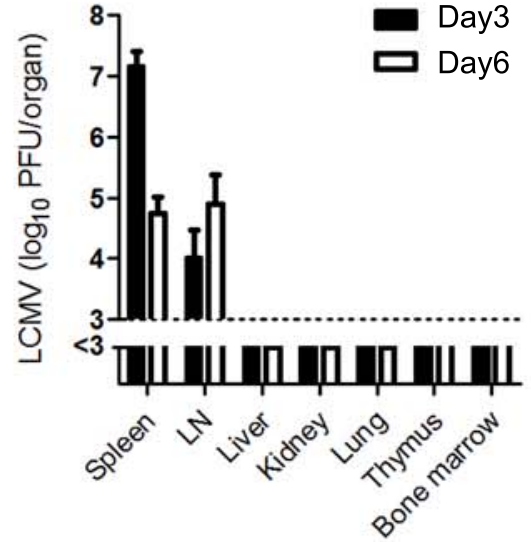


Figure 2
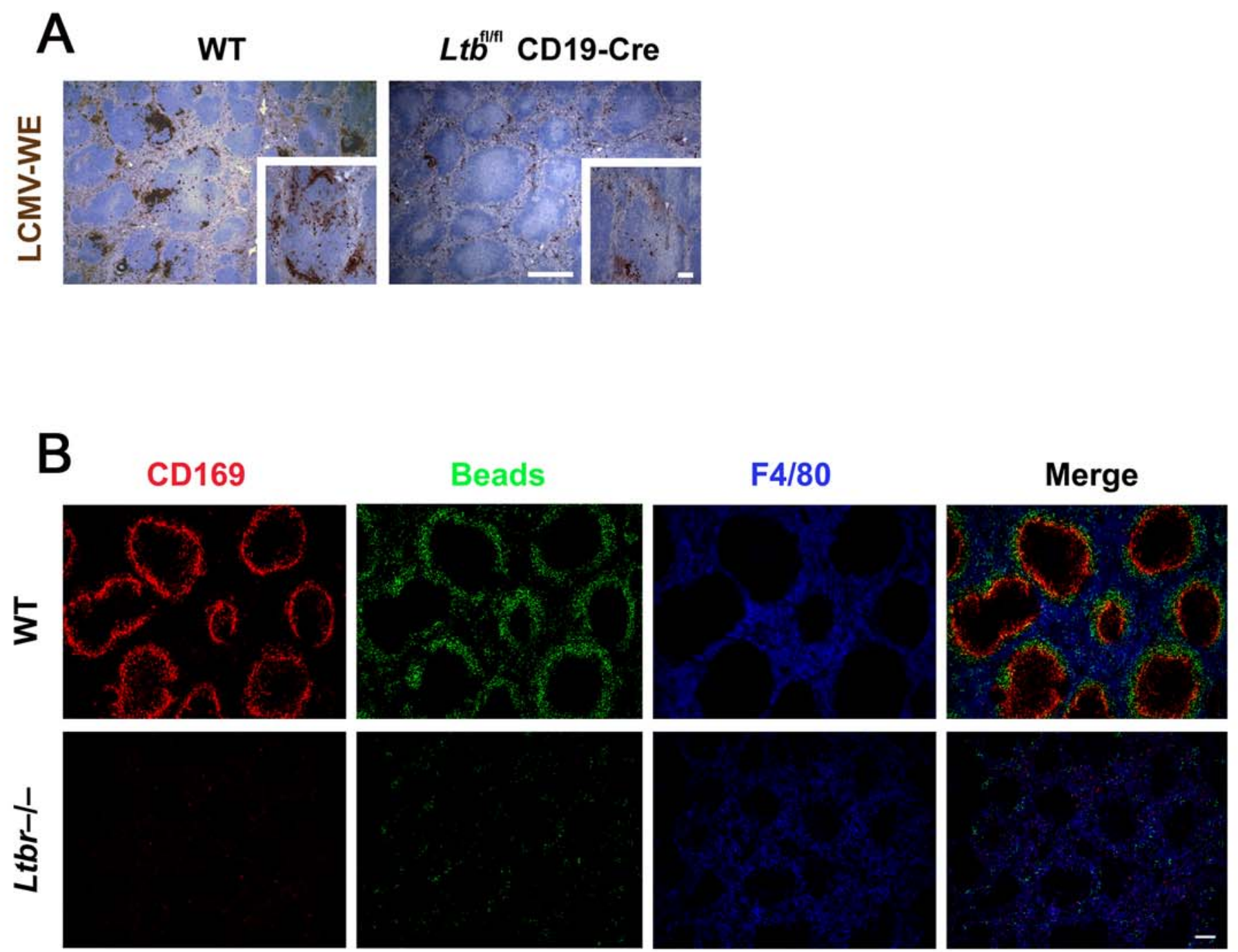

C

Spleen

Ltbr

WT

Ltbr ${ }^{-1-}$

WT

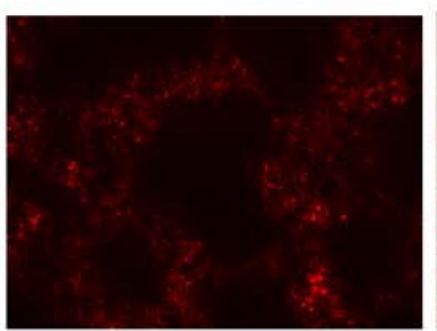

Liver
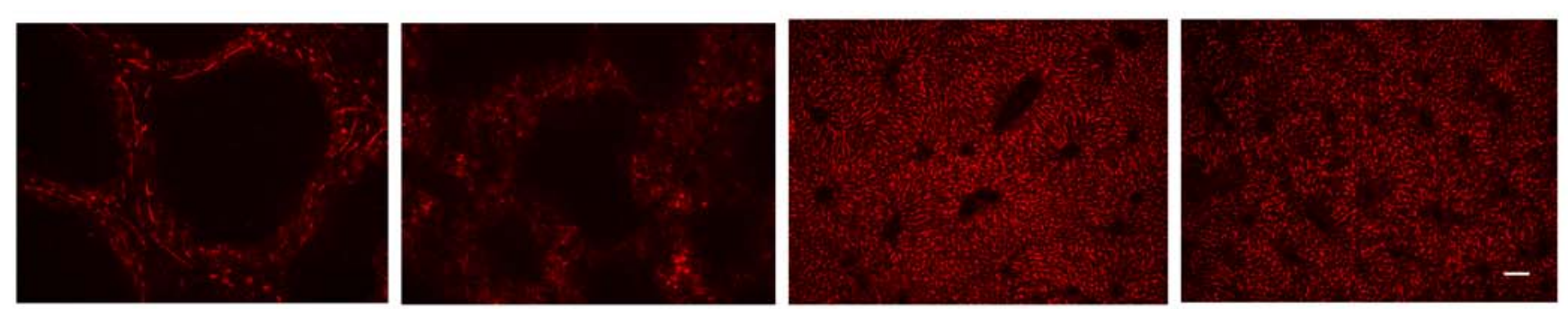
Figure 3
A
B
C
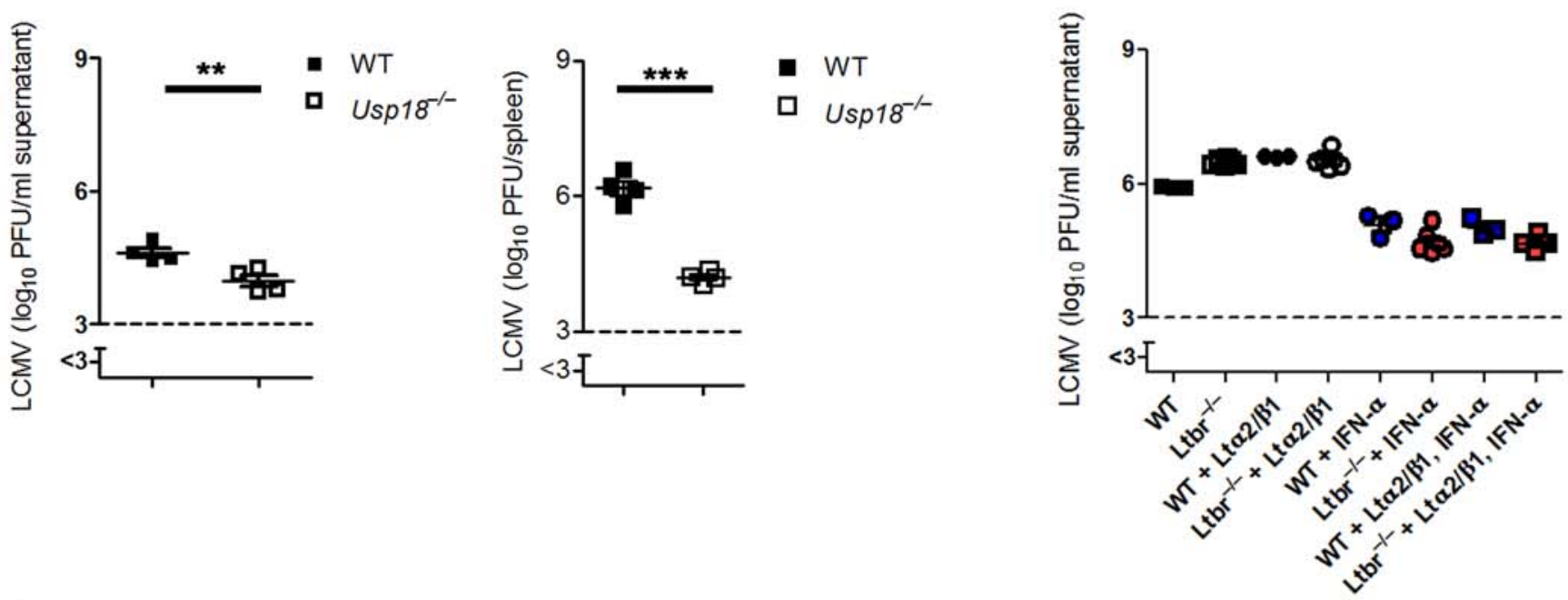

D

E
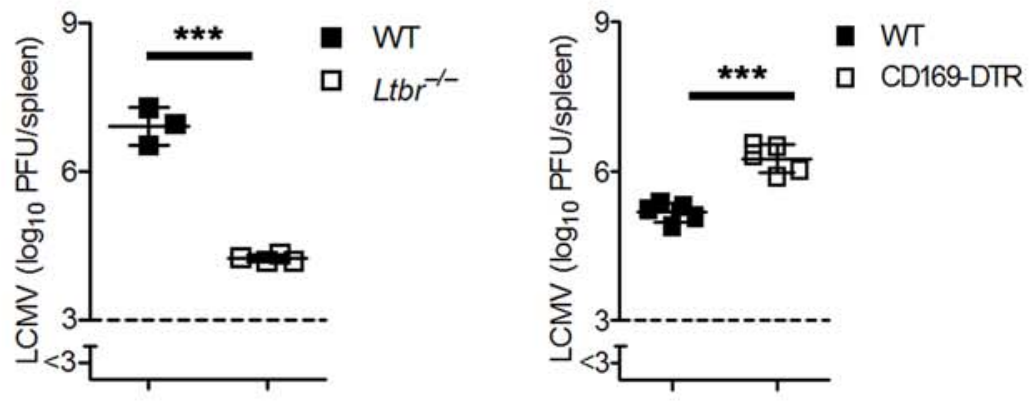

F
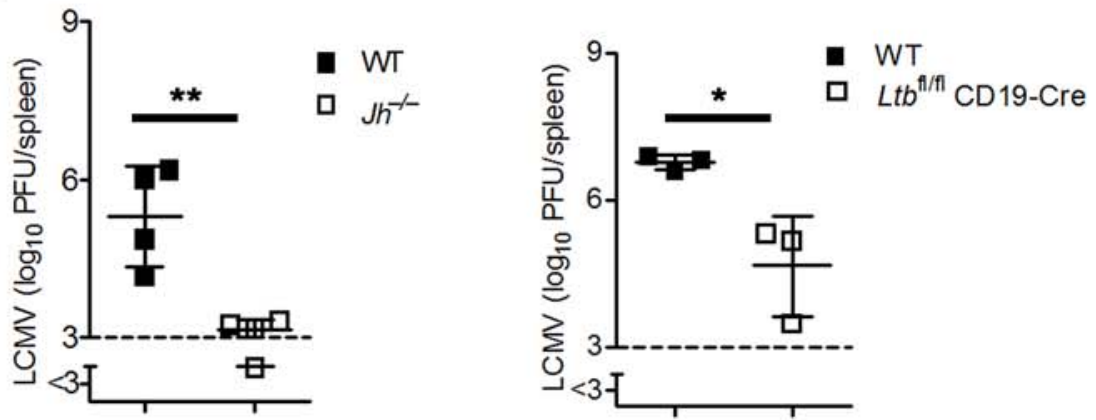
Figure 4

A

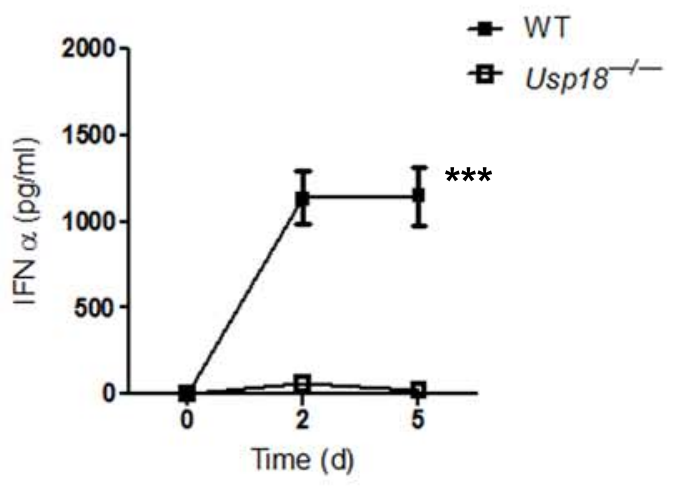

C

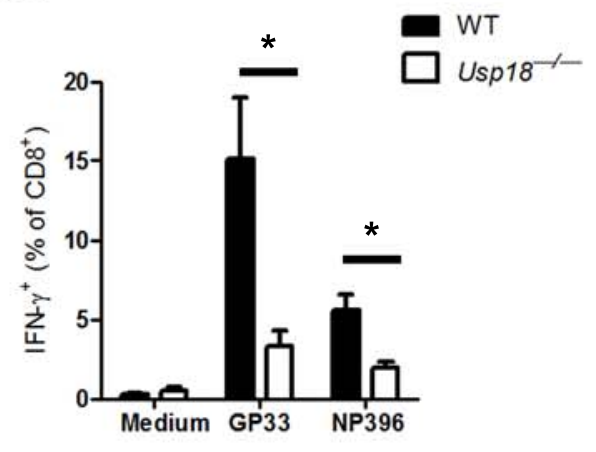

B
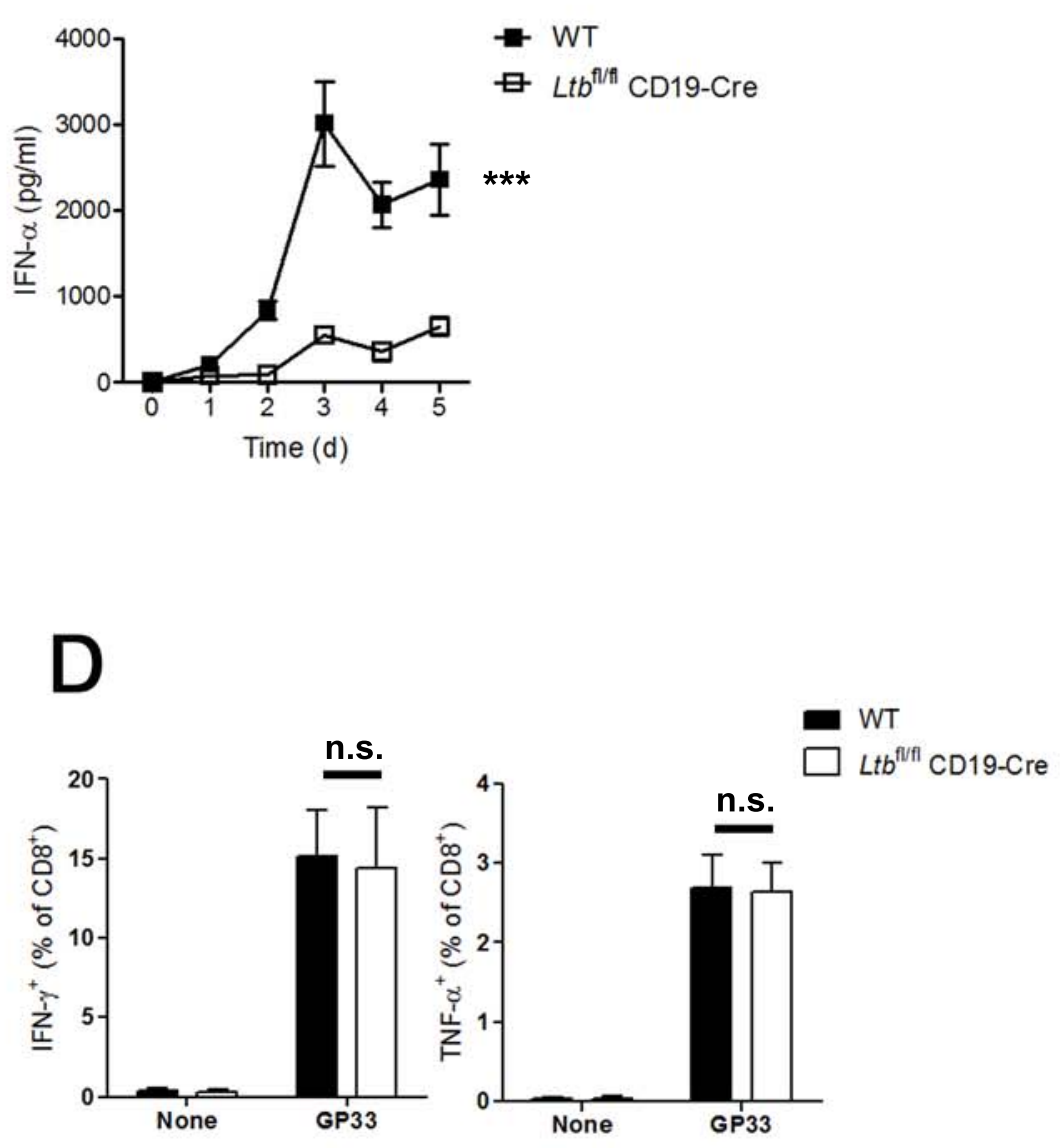


\section{A}

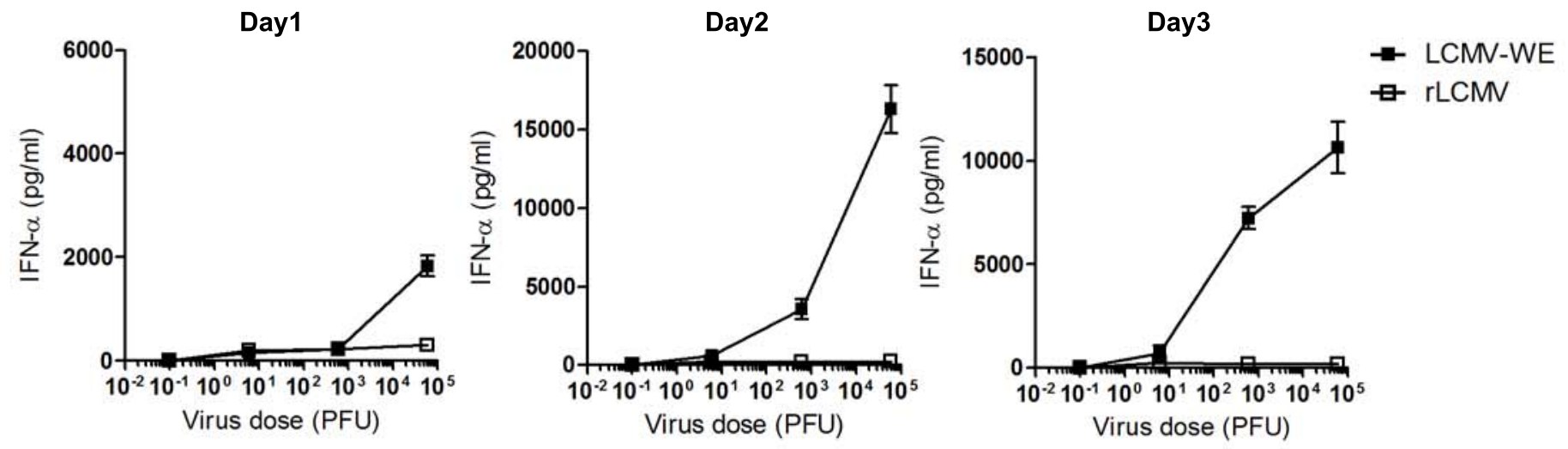

B
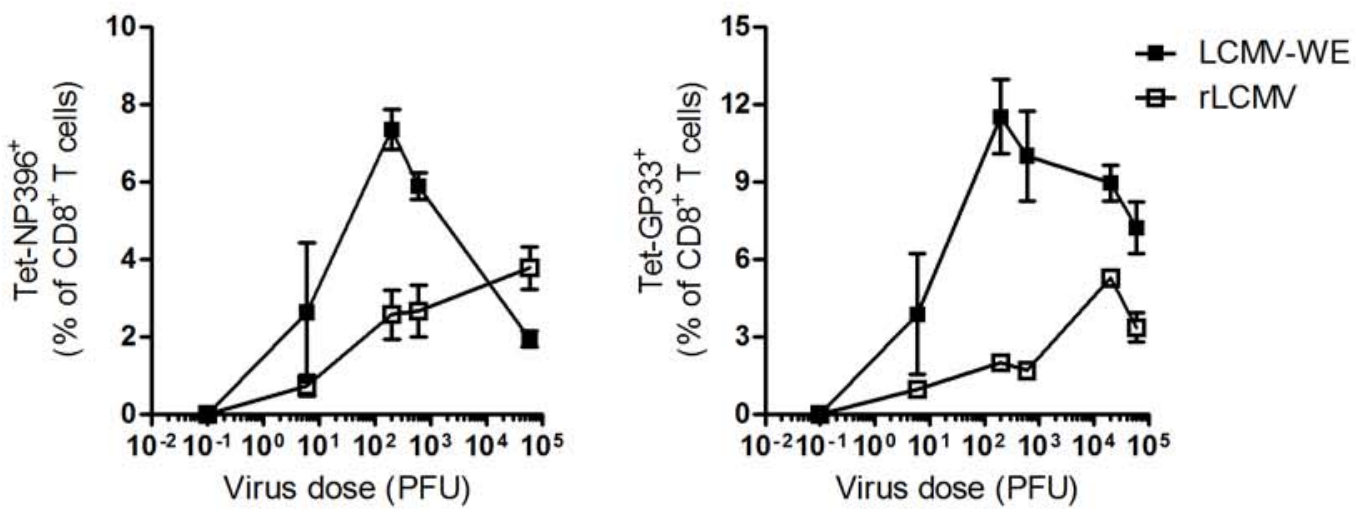

C
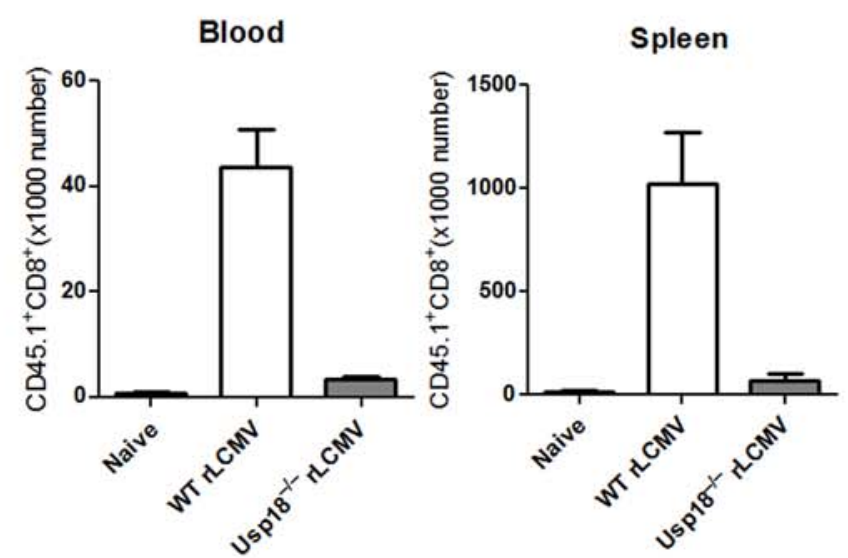